\title{
Strings Attached? How Global South Partner Organizations' Perceptions of Feminism Shape their Relationships with Feminist Foreign Policy Donors from the Global North
}

\author{
Tiffany Laursen
}

\section{Introduction}

As part of a larger global movement to promote gender equality and women's empowerment (GEWE), governments have increasingly tied their bilateral foreign aid agendas to feminist foreign policy (FFP). In 2014, Sweden was the first country to proclaim that its bilateral foreign aid policies would promote feminist ideals. In 2017, Canada announced its Feminist International Assistance Program (FIAP) would focus on eradicating poverty through promoting shared values by committing no less than 95 percent of Canada's bilateral international development assistance would "target or integrate gender equality and the empowerment of women and girls" by 2021-2022 (Global Affairs Canada 2017). More recently, France, Mexico, and Spain have committed to FFP while the United Kingdom and Australia have revamped their focus to align with FFP. Moreover, Iceland, Ireland, Belgium, and the Netherlands have committed more than 60 percent of aid to FFP/ GEWE without a formal declaration (OECD 2018).

Although a welcomed commitment to advancing equality and opportunities for women and girls, some critics claim that FFPs (particularly FIAP) contain vague goals and outcomes (Morton/Muchiri/Swiss 2020) while instrumentalizing women and girls (Tiessen 2019). Further, they proclaim the need to utilize more innovative approaches when translating policy into action (Rao/Tiessen 2020). Other critiques warn of the importance of understanding the plight of women and girls in their context without importing western ideals (Robinson 2021) while also considering the diverse views and locally-based approaches to feminism (Rao/ Tiessen 2020). The cautionary advice recognizes the history of the various forms of feminism originating in the Global South. In Africa, for example, Mama (2004) identifies the roots of feminism trace back to the $18^{\text {th }}$ century (and potentially earlier), while more modern approaches based their activism on an African agenda set in 1977 by the founding of the Association of African Women for Research and Development (Ampofo et al. 2004). More broadly, Global South feminist activism has largely coincided with political public discourse around post-independence nation building and globalization (Mohanty 1984; Ampofo et al. 2004). National movements shaped the way feminists in Africa and Asia framed their discussion points away from domestic violence and reproductive rights opting instead to 
center their advocacy on "militarism and health, to environmental and economic justice, social development, human rights, and population” (Antrobus 1996: 64).

Another aspect of Global South feminist advocacy was the erasure of "global sisterhood" which asserted women's oppression was solely related to the commonality of their gender. Theorists instead offered intersectionality as a guiding lens for development programming, which recognizes compounding effects of multiple variables including class, race, and ethnicity (among others) in the struggle to obtain social justice for women (Mohanty 1984; Antrobus 1996). Intersectionality includes diverse perspectives that are reflective of a variety of local contexts. Without recognizing the variations and contextually relevant voices, one risks assuming that all women - particularly all women in the Global South - have the same interests, challenges, and agendas (Mohanty 1984; Win 2004; Okech/Musindarwezo 2019). Narayanaswamy (2016) takes this one step further by explaining that even the more recent effort to create spaces for less dominant voices from the Global South is limited by the small representation of Southern feminist voices and the narrowly defined conception of feminism, joining the call for a greater emphasis on locally-based feminisms (Moghadam 1998).

To more fully include the multiplicity of voices and perspectives of diverse feminisms rising from the Global South, there requires a rejection of a hierarchal ranking of dominant forms of feminism (often associated with Western feminism), and an inclusion of more peripheral interpretations of feminisms (Lal et al. 2010). Decentering Western feminism and embracing diverse feminisms paints a more accurate picture of local agency whereby the wide array of Southern voices are viewed as "fully capable of accurately interpreting their complex reality, rather than [Northern scholars] attempting to represent their perspectives on their behalf" (Tiessen/Lough/Cheung 2012: 138). This guarantees feminist knowledge is not only reflective of the numerous backgrounds displaying diverse voices, but also allows the re-centering of knowledge to include the voices in the various regions of the Global South. Bringing in the voices of people who have otherwise been overlooked or silenced is not only necessary but also invaluable in guaranteeing the efficiency and positive influence of FFP on livelihoods.

\section{Literature Review: Links between Policy, Funding, and Implementation}

The framing of feminist agency becomes a pivotal intersection for FFP and GEWE initiatives laying between public policy (e.g., FIAP, Sweden's feminist foreign policy), NGO funding initiatives, and transnational actors including international development volunteers and partner organizations in the Global South. In this juncture there is an ongoing process of negotiations, where the values and priorities of multiple stakeholders come together to make decisions on program funding 
and thereby development. Theorizing relationships between NGOs and governments, Coston (1998) describes a continuum between asymmetrical power based on resistance to pluralism (isomorphism and dependence) characterized in three ways (repression, rivalry, and competition), and symmetrical power based on acceptance of pluralism characterized in five ways (contracting, third party, cooperation, complementarity, collaboration). Simply, organizations will push against donor-imposed initiatives to varying degrees as they strategically navigate their social mission objectives. Empirical analysis show many Global South organizations conform while some are able to resist donor agendas (Rauh 2010; Lefroy/ Tsarenko 2013). Moreover, promoting local initiatives through network support requires rethinking "the link between policy and implementation, recognizing that both are political processes and that while policies set agendas, both policies and their implementation are deeply influenced by societal factors" (Parpart 2014: 382). While FFP agendas and GEWE programs are largely shaped by societal factors, the norms and values that shape the application of feminist ideology between donors in the Global North and implementers in the Global South may vary significantly.

Sundstrom (2005: 422) expands this argument to consider the way foreign aid is used to promote universal norms and the limited impact these universal norms have on a "successful NGO movement."

To add to this framework, it is important to move beyond the binary of "conformity" and "resistance" to consider the process of negotiation, partial adaption, and diplomacy. Building on this analytical lens, this paper draws on interview data from ten countries in the Global South. This paper explores Global South partner organization's perspectives on how the emergence of FFP from the Global North has shaped their relationships with donors as well as the programs that support local beneficiaries. Exploring how FFPs play out in practice, several questions guide the analysis: are donor initiatives known to partners? Do Global South partner organizations integrate feminist priorities into their programs to obtain funding? Do donor approaches resonate with their own feminist/development priorities - why or why not? What is the perceived value added, if any, of FFP?

\section{Methods}

Interviews were conducted in 2018 - 2019 with 150 partner organization staff in ten countries. Once all interviews were transcribed, data were coded to identify common themes throughout. Data were analyzed using discourse analysis. The introduction to this special edition has more detailed information on the methodology for data collection and the analysis of findings. 


\section{Findings}

The questions outlined above were examined in the interview transcripts using discourse analysis. Each of the scripts were open coded using Atlas.ti.9 software. The following themes emerged as predominately discussed among all interviews: variation in the level of awareness of FFP, variations in the level of utilization and integration of FFP, variation in perception of congruence between Global North donor agendas and Global South priorities, and the distinct contributions donor policies have made to GEWE. As each theme was explored, the overall findings reveal that partner organizations in the Global South pursue partnership-style relationships with NGOs in the Global North where autonomy and cultural relevancy are valued as organizations navigate FFP.

\subsection{Awareness Levels of FFP/GEWE Tied to Donor Agendas}

During interviews, partner organization staff were asked whether the donor policies guiding the work of their Global North NGOs partners were known to them. Particularly, partner organization staff were asked whether they had heard about FFP. Further, we inquired about how international feminist policies like FIAP are guiding development work in their country through specific channels such as Canadian NGO collaborations and the role and impact of transnational actors such as IDVs. The degree to which staff were aware of FFP ranged from those who have never heard of FFP to those who are well versed and actively implementing projects. Those who have never heard about FFP were aware of feminism more broadly: "We have not heard about the policy in Sweden or Canada, but we are aware about the feminist perspective and how it works in general" (Nepal). Some were unaware of policies because they had not investigated it: "No. I could have heard, but it's not something I really dig into" (Tanzania).

Several interviewees commented on their knowledge of FFP but were unaware of the particulars, such as in Ghana: "Not the details of them but I do know the existence." Moreover, some interviewees tied their limited knowledge to having GEWE in their periphery resulting in lack of awareness, as in Vietnam: "I heard a bit about that, but I didn't really do a lot of research... We have been running a few projects on gender equality promotion, but it doesn't mean that we are very strong in women's issues." Still, others were aware and have studied FFP, like in Uganda: "I've read about them...They have a document about international relations in respect to gender." Still, others were aware because they are required to report outcomes: "We are familiar because it [is] something we have to show on the proposals" (Tanzania). 


\subsection{Utilization Levels}

Corresponding to level of awareness, utilization of FFP funding ranged between those who do not utilize it in any way and those who do. Those who do not utilize the donor initiatives noted their reasoning was based on either their lack of knowledge of opportunities or their reliance on other sources of income: "We do not receive the budget from the government, we are funded by products and services" (Vietnam). However, several partner organization staff stated their level of utilization is tied to the organization remaining autonomous: "Decisions need to be made by us. We are happy to talk about things, but ultimately, we make the decisions about what we think is best for us" (Guatemala).

Several study participants presented examples of not applying for funding because of the inability to meet donor's requirements. This was largely a concern when donors required quotas for a specific number or percent of women to be involved in the implementing organization: "We couldn't even apply for that funding because one of the requirements was that at least $50 \%$ of the leadership of the organization should be women, and ours was 40\%" (Tanzania). Partner organizations also discussed the requirements for women's participation in organizational leadership positions: "We were looking for a communications person and wanted a woman. It was very difficult; we couldn't reach our goals. Sometimes, when you come to the effectiveness and efficiency, it's not easy to get [70 percent women]" (Tanzania).

In their quest to build capacity, another frustration came from requiring femaleled initiatives when women were not trained first. Interviewees from Malawi elaborated on this point noting that women need training to be equipped to take on leadership roles. The interviewee described examples of donors funding women-only projects that resulted in unsuccessful programs because the women were "not well qualified to handle the project."

Funding tied to women in organizational leadership was seen as having potential for opportunities if donors are willing to be flexible. A participant from Tanzania noted the significance of flexibility in funding arrangements so they can more effectively use the resources to "improve the gender relations in our organization, but also to improve the capacity of women to take more responsibilities throughout the organization." The participant went on to explain that without flexibility in the funding model, the organization is unable to build the capacity of the women to implement the project.

Other interviewees took this sentiment a bit further. They described a negotiation process weighing the interests between donor objectives and project outcomes that involved strategies to get women into leadership positions. This negotiation process fills priorities for both donors and the organization "when we post a woman 
from that level to higher level, we fulfill the donor's interests. But we finish with our interests by getting women and men on the common ground" (Tanzania).

Out of these requirements for GEWE targets, some partner organizations expressed that their priorities have to be adapted to survive: "The world has changed and most of the organization or countries are focusing on providing more knowledge to women and youth. Without the organization shifting, it's going to be very difficult for an organization to be stable" (Tanzania). One interviewee suggested shifting requirements away from organizations and towards the beneficiaries, thereby building capacity for future leaders: "If we empower girls [beneficiaries of our services], then we will be able, later, to get them into leadership. But...if the policies don't target the beneficiaries, then you won't reach this 50/50 in decision making" (Tanzania).

Even if partner organization staff were unsure of their capacity to meet donor requirements, some have taken up the challenge and applied for funding. They used the opportunity to assess their situation prior to accepting investment, particularly when the money has been tied to increasing the number of women participating in projects as in Tanzania: "We had one week ...to start planning, to [send] information, so [we could] get [minimum of $60 \%$ women] ...to attend that workshop. We did that and we saw it was something we can now manage...So we accepted the proposal" (Tanzania). (See Nguyen's article in this collection for further discussion on mainstreaming gender priorities).

Given their utilization strategies, partner organizations found ways to incorporate gender requirements into their programming as described by an interviewee in Ghana: "If you are doing training, for instance, then you have to figure a way out to add gender equality component to it for it to qualify for funding... to receive funding from FIAP." However, it appears that much of the interpretation of donor requirements involved increasing the number of women participating. Still, some organizations do not just pad their training with increased number of women to obtain funding; they are actively engaging and promoting initiatives: "Thanks to these policies from other countries, we've gotten funding for organizations to protect women and I think this has improved a lot" (Guatemala). The improvement (interpreted as stemming from Canada's FIAP) was noted as a welcomed change for women, as one Ghanaian interviewee illuminated: "Women have always been on the wrong side of things for years so there's nothing wrong with [trying to] balance the equation." 


\subsection{Perceptions of Coherence of FFP/GEWE Between Donors and Partner Organizations}

Beyond understanding levels of awareness and utilization, we were also interested to know the level of similarity between partner organizations and donors' perceptions of feminism, FFP, and GEWE. We specifically asked if FFP has changed the way services are planned and provided to the beneficiaries. Some interviewees expressed concerns with donors that attach strings to their initiatives because GEWE initiatives left men and boys out of capacity building “....when a feminist is leading this type of work, they insist on the feminist approach and then they leave out the men..." (Malawi). Specific examples from Malawi demonstrate the need for a broader approach to community development that promises buy-in from all members of the community. This should particularly include men and boys since they can act as the 'gatekeepers' and limit or reverse program success. In their experience, GEWE programming targeting only women and girls results in spoken and unspoken backlash. One interviewee described a scenario where micro finance programs designed solely for women failed to gain buy in from men who in turn "are the ones [that] are going to frustrate these kinds of efforts." This interviewee further described a sabotaging effect in a scenario where girls were given scholarships for secondary education, but when the community was surveyed, the organization found that instances of early pregnancy rose while instances of dropping out of school had not changed. Upon inquiring, the communities expressed that some of the boys were poorer than the girls who received scholarship. In turn, the boys got frustrated and focused on disrupting girls' education. The interviewee concluded with one important, albeit hard learned, lesson: "If you don't build capacities in families and communities to handle these issues even if you finance these initiatives, sometimes they don't yield the results you expected because Malawi is not Sweden, Malawi is not Canada." (See Shahadu Bitamsimli's article in this collection for further discussion on including men and boys).

An adapted strategy between donor initiatives and community objectives was raised in a conversation about how to tackle root causes of gender inequality. A Nepalese interviewee explained the relationship between economic status and domination as a primary root cause of gender inequality: "If we cannot provide access to resources and information, then this situation cannot be changed by lobbying and advocacy...If ...you have access to resources, nobody can dominate you. Whether you are female or part of another socially excluded group."

In accordance with addressing root causes of inequality, it appears there is a perception that donors lack understanding the reality of country contexts. Some interviewees suggested that donors have vague end goals that they struggle to tie to community objectives. The struggle for donor relevancy was perceived by some partner organization staff to stem from inadequate vetting as donors post requests 
for proposals and then evaluate writing skills as opposed to building relationships with partners who are intermediaries to the beneficiaries: "A best writer is not the right partner... Donors should change their modality; they should approach the partner directly. They should build capacity of the partner, but also go directly to meet the beneficiaries through the partners...” (Tanzania).

Staff from partner organizations considered the misunderstandings between themselves and donors explaining that donors were "not informed... every donor needs to know more" (Tanzania). Moreover, interviewees used the perceived disconnect to invite donors to their communities: "They should come on the ground [and ask], 'what do you need? What do you need for the coming year? What do you need from me?" (Tanzania). This approach to assessing needs would begin to address misunderstandings.

In a striking critique of the disconnect between cultural context and donor understanding, a broad concern emerged that contingency aid takes advantage of beneficiaries. One Malawian interviewee expressed: "Attaching such kind of conditions to aid I feel [is] just taking advantage of the poverty that people have. People are poor and we should not attach conditions to assisting the poor." Seemingly tied to this ideology (and that of the concerns raised above) was an overwhelming reiteration that organizations will not accept financial assistance that does not align to their objectives - appearing to solidify the autonomy of partner organizations: "If we were to accept [funding] then it means that it aligns with what we are already thinking or the policies that exist in this country" (Ghana).

In this alignment, many interviewees expressed their commitment to global causes including GEWE - sometimes expressing their own expertise. In Senegal for instance, one participant stated, "we specialize on these issues. We have our terminology that does not differ too much from what is being done at the global level." Further, many partner organization staff expressed that FFP is helpful to their initiatives "even before you ask whether we shall align, we are already aligned and as such any other organization that will come in to fund such a program will just be enhancing a lot of value and improving human dignity" (Kenya). Beyond alignment, organizations expressed their guiding principles are not negotiable and are the bedrock of their organizational permanence: "If we were not able to follow the policy, we have created for ourselves, we would be nothing." (Tanzania). Another interviewee in Tanzania asserted, "because we are not against [GEWE]... your support cannot really dismantle our framework... We will not allow that. But we don't show it, we persuade them."

Calculated decision-making seemed to be a strategic undertone. One Guatemalan interviewee described 'collaborative wins,' seeking funding that is a "win-win situation for us. For example, a Guatemalan congresswoman wanted to give us funding 
recently, and it was going to be good money, but the things that she wanted to do were not worth the money."

Beyond mutual wins, interviewees described a vetting process. The process reflected careful decision making that challenges the notion that civil society organizations (particularly in the Global South) will desperately accept funding regardless of goal alignment. Instead, as exemplified by a Tanzanian interviewee, the vetting process starts with understanding criteria, thematic areas, and how the funding objectives are in line with their work in the communities: "Before writing a proposal ...we consider...three things: the demand of the community, the demand of donor, but also the demand of the government by using existing policies, strategies, plans, laws... to ensure that [every component] ...complements each another."

To summarize, many interviewees did not believe that donor policies changed the work they did largely because: "Our mission is already deeply rooted in women's... empowerment” (Guatemala). Moreover, interviewees asserted selection bias when discussing their strategic responses to donor calls for proposals: "We don't respond to every call, but we fix to our mission and objective of the organization" (Tanzania). Selection bias gives further evidence that organizations in this sample are autonomous entities, free of dependency. Emerging from the data are an abundant amount of evidence that interviewees possess an embedded sense of ownership over their own initiatives:

The funding that are being delivered from outside must meet our demands. The original idea must be borne by us...developed by us and what we believe in. Imposing an external influence... we honestly don't entertain that... We existed for more than 6 years without external support (Tanzania).

In conjunction with importance of local ownership, interviewees provided strong evidence that context matters. A large concern was FFP originating in the Global North does not translate well when applied to Global South countries. As a Ghanaian woman proclaimed: "I am an African. And African women - our cultural background is different than somebody from Sweden.” Another Ghanaian interviewee clarified: "Development support that requires us to adjust into feminist policies that are not in sync with our culture or that we find that doesn't bring in positive benefits to us is something that we will contest strongly" (Ghana). Interviewees provided examples of nuanced differences that effect programming - even within communities that are part of one country. In Tanzania, for example, an organization attempted a women's empowerment project with keeping chickens. However, in the local tribe, women were not permitted to have chickens resulting in failure: "When I came to encourage them or tell them to keep the chicken, it was difficult [for them] to understand my idea." 
Beyond nuances, a larger discussion of context surrounded the legal translation of FFP, specifically when discussing LGBTQ+ and abortion rights. In countries where LGBTQ+ and abortion are illegal, some organizations expressed their decision to honor the legal system in their country for their own organizational survival: "LGTB is illegal... it can be a personal trait which we don't have any problem with, but we as an organization we have to follow the government regulations. If we go against it, it can be a disaster" (Tanzania). While some organizations honored laws, others found ways to navigate and/or compromise their practice to promote ideals like in Senegal where abortion is illegal: "Because of a strong lobby, we cannot work openly on [abortion], but must find strategies to strike a balance... with all the consequences resulting from women being raped, or who are subjected to incest and become pregnant...we look for solutions." In this compromise, however, organizations expressed that they risk not only legal repercussions but also future monetary support: "There are partners that finance you and then they hear that somewhere you are negotiating financing with people working on the issue of abortion, they can withdraw their financial support" (Senegal). In both examples, organizations strategically navigated their work, describing themselves as a liaison between donors and the communities they work. Interviewees confidently expressed their conviction that receiving money from a feminist-focused donor (such as FIAP) will not change their work because: "We work in the communities, so we know what is best for the communities and I believe we are in a better position to talk to any donor and see their terms and conditions.” (Uganda)

Regardless of the limitations, partner organizations gave advice to their donors:

Whatever you do, if you're planning to implement an activity, [ask] what kind of legal management activities it has? For example, if you're doing economic empowerment, what are the policies in Tanzania that are dealing with economic empowerment? What are the policies, procedures, and administration on the ground are dealing with economic empowerment? What are the conventions, statements, and policies at the international level which are dealing with economic empowerment? How are they fitting up with your implementation at the local context? (Tanzania)

\subsection{Unique Contributions of FFP/GEWE Donor Initiatives}

Interviewees offered their observations about the unique contributions that FFPs have provided to programs. Several commented that donor initiatives force governments and organizations to think: "I think they push our country to think in different ways" (Guatemala). Another interviewee expressed: "When [the government] gets aid like that it forces the government to think...unless if they don't need the money then can say get your money and go out" (Malawi). In Uganda, an interviewee described receiving funds as an opportunity for growth: "I think 
it's an opportunity because if in our country we don't embrace feminism, I think they are going nowhere." The interviewee continued to describe their perception of the patriarchal society where male-dominated leadership makes decisions that maintain inequality through laws and limited funding. Moreover, this interviewee offered a solution: "If we had more women, things like more funds would be allocated to the education system and changing rules for girls to stay more in school."

Several interviewees described receiving money tied to GEWE as strengthening, maximizing, or enhancing their work and their global relationships: "[Receiving] funds [from] a government that has declared itself as feminist... strengthens us because it also improves the relationship" (Peru). In Kenya, an interviewee extended this notion by stating: "[Funding] such a program... [is bringing] a lot of value and improving human dignity and especially to the disadvantaged, to the most marginalized."

Overwhelmingly, interviewees described their relationships with donors as a strategic partnership: "We are not normally asking for help, but it is rather partnership and cooperation. We do plan together and do together" (Tanzania).

\section{Analysis}

The findings reveal the relationship between FFP, NGOs based in the Global North, and partner organizations based in the Global South consists of negotiations with important nuances in the management of competing priorities. Partner organizations highlight a range of responses to external funder priorities including acceptance, conciliation, compromise, resistance, and rejection. Decisions pertaining to partnerships with donors and implementing Global North NGOs (and the transnational actors that work in-between Global North NGOs and Global South partner organizations) reflect partner organization agency in decision-making, their strategies to navigate initiatives, as well as local priorities and cultural realities. Two common themes emerge from these findings: calculated decision making and discussion of shared values. This section considers these two findings and the distinct strategies employed to navigate these themes.

\subsection{Calculated Decision Making}

Admittedly, many of the interviewees in the sample are associated with feminist ideals, however, their candidness leads to a conclusion that while there are at times perceptions of imposed ideas/norms (be it from FFP originating in the West, or international development volunteers who bring their own culturally bound gender norms), partner organizations navigate, negotiate, and may ultimately resist forms of imposition. Their calculated decisions about the extent to which funding is needed and valued in relation to their own priorities, principles, and capacities 
is central to their strategic approaches. Partner organizations navigate structural inequalities while also exerting their own agency. For example, partner organization staff underscore the significant structural inequalities in which they must make decisions such as donor driven agendas. The example noted above pertaining to FFP being linked to program priorities has the potential to influence program commitments of partner organizations who are navigating systems of inequality with the purpose of addressing extreme poverty. Strategies identified by partners to mitigate or disrupt these structural inequalities were noted. Recommendations included increased commitment by donors to listen to local perspectives and inviting donors to visit the country and communities in which they live and work. Greater donor interaction is viewed as a pathway to fostering a stronger voice and improved partnership arrangement so that FFP initiatives better reflect local realities. The partnership-style approach allows for negotiating funding terms and is a strategic measure used to keep the interests of local governments, communities, and organizations at the center of initiatives. Improved relationships that allow and respect diverse perspectives between donors and partner organizations are instrumental in combatting Western imposed or donor-focused priorities. NGOs from the Global North that collaborate with partner organizations in the Global South could play a bigger role in advocating for increased donor responsiveness to local priorities. The mechanism to achieving this objective might involve the uniquely placed transnational actors working as IDVs. IDVs work closely with partner organizations and communities and through this sustained collaboration, observe the way that partner organizations navigate and translate FFP and GEWE priorities into relevant and accepted policy and practice within the partner country. IDVs can use this knowledge to inform - and to lend support to the voices of partner organizations in championing their priorities - to Global North NGOs and donors.

\subsection{Discussion of Shared Values, Universal Norms, Local Manifestations, and Agency}

Overwhelmingly, these data reinforce the significant ideal that 'context matters' in considering GEWE as a universally-shared value. The concept of universal norms and/or shared values is clear: people - regardless of culture - value and respect women and girls. The cultural context of GEWE, however, seems to be complicated with nuanced norms. Universally embraced norms may be that women are valued in societies, however, throughout the interviews it was clear that universal norms do not manifest themselves in all cultures the same way. Interviewees described the nuances in various examples: culture, laws, and LGBTQ+ rights; culture and funding girls' education and/or women's microenterprise at the expense of men and boys; creating spaces for women as professionals while also valuing and respecting the culturally coveted role as family nurturers (an interviewee explaining that Nepali women are given permission to leave work early to attend to family 
matters, for example). The position and status of women in society - including the way women experience respect, reverence, and subjugation - is more complex, nuanced, and related to context. The complexity stems from the nuances that are related to context (including law and culture which deserve separate description and analysis but expands beyond the scope of this paper) and is essential to the development and implementation of relevant GEWE programming. Local partner organizations with direct knowledge of these nuances strategically negotiate interests, attempting to find middle ground between donors' initiatives, beneficiary needs and cultural context, and local government laws and agendas. Although universal norms position organizations to accept funding, the nuanced differences create the spaces that open the door for negotiation. Partner organizations described continual negotiations with their donors to find common ground; therefore, the various manifestation of cultural values and the agile and diplomatic skill set of partner organization staff shape how FFPs play out in local contexts.

The variance in manifestations is where local culture and agency are central players in deconstructing local systems of oppression - as they see fit - which may differ from dominant perspectives, particularly those from the Global North. One example is the discussion on goals and outcomes. Scholarly critiques note that FIAP has vague goals (Morton/Muchiri/Swiss 2020), but interviewees in this study perceived donors' goals to be too focused. Staff from partner organizations expressed concerns that donor objectives that are too narrow may disqualify partner organizations from funding opportunities which could be used as a steppingstone to promote transformational change towards equality. The prominent example given was requiring a certain number of women to be in leadership positions to apply for funding. While vague goals hold organizations less accountable for specific outcomes, they also allow local initiatives more freedom to adapt programs that fit their local context and needs. Ambiguity may then provide time and space to train women appropriately and better position them for future success. In the example of promoting women to leadership, local manifestations could benefit from vague goals to obtain funding - fully recognizing that transformational gender equality requires more than counting the number of women in leadership positions. The onus would be on the partner organization to outline specific goals to train women for leadership - or a more locally relevant GEWE initiative - and to show the milestones for how the longer-term process of transformational change could occur. More generally and beyond the example of women in leadership (which admittedly is one small prong of a feminist approach which has a larger goal to promote transformational change), shouldn't the development field seek to allow donor funding that perhaps is fuzzier, but also petitions for context specific outcomes in projects that address local needs related to GEWE? Is that not the promise of accepting feminist multiplicity and embracing agency? 


\section{Conclusion}

As Global North governments have increasingly tied their development aid to feminist foreign policy, Global South partner organizations have responded using many diverse strategies, (including, but not limited to, compromise and conciliation) while the recipient communities range in their response between acceptance and resistance to GEWE programming. The range of strategies utilized by partner organizations expand the conversation beyond Coston's (1998) discussion of push back by civil society organizations as well as Sundstrom's (2005) argument of promoting universal vs. local norms and impacts on NGO movements. Instead, partner organizations strategically navigate the funding mechanisms and priorities in relation to their ability to utilize funding in line with their own priorities, particularly when identifying community-level competencies and capacities to meet donor requirements. Many partner organizations welcome these opportunities, viewing them as openings for negotiating partnership style relationships. In this interaction, partner organizations in the Global South have maintained their autonomy and cultural relevancy, while also promoting GEWE initiatives in their countries - and specific cultural contexts. Greater flexibility among donors to allow local initiatives - and thus agency - to be at the center of development is central to recognizing locally-based feminisms. There may be a role for transnational actors such as IDVs working in-between the spaces of Global North NGOs and partner organizations who can better advocate for change at the policy level without compromising the autonomy and agency of partner organizations. As more countries and NGOs tie their funding to GEWE objectives, it is imperative to continue to "check in" with the level of autonomy partner organizations experience to maintain local relevancy, agency, and the expression of distinct and culturally specific feminisms.

\section{Bibliography}

Ampofo, Akosua Adomako; Beoku-Betts, Josephine; Ngaruiya Njambi, Wairimu; Osirim, Mary. (2004): Women's and gender studies in English-speaking sub-Saharan Africa. A review of research in the social sciences. In: Gender and Society, vol. 18, no. 6, pp. 685714.

Antrobus, Peggy (1996): Bringing grassroots women's needs to the international arena. In: Development, vol. 39.

Coston, Jennifer (1998): A model and typology of government-NGO relationships. In: Nonprofit and Voluntary Sector Quarterly, vol. 27, no. 3, pp. 358-382.

Lal, Jayati; McCuire, Kristin; Stewart, Abigail J.; Zaborowska, Magdalena; Pas, Justine M. (2010): Recasting global feminisms: Toward a comparative historical approach to women's activism and feminist scholarship. In: Feminist Studies, vol. 36, no. 1, pp. $13-39$.

Lefroy, Kathryn; Tsarenko, Yelena (2013): From receiving to achieving: The role of relationship and dependence for nonprofit organisations in corporate partnerships. In: European Journal of Marketing, vol. 47, no. 10, pp. 1641-1666. 
Moghadam, Valentine M. (2002): Feminisms and Development. In: Gender \& History, vol 10, no. 3, pp. 590-597.

Mama, Amina (2004): Demythologising gender in development: Feminist studies in African contexts. In: Institute of Development Studies Bulletin, vol. 35, no. 4, pp. 121-124.

Mohanty, Chandra Talpade (1984): Under Western eyes: Feminist scholarship and colonial discourses. In: Mohanty, Chandra Talpade; Russo, Ann; Torres, Lourdes (ed.): Third World Women and the Politics of Feminism. Bloomington, pp. 51-80.

Morton, Sam; Murchiri, Judyannet; Swiss, Liam (2020): Which feminism(s)? For Whom? Intersectionality in Canada's Feminist International Assistance Policy. In: International Journal, vol. 7, no. 3, pp. 329-348.

Narayanaswamy, Lata (2016): Whose feminism counts? Gender(ed) knowledge and professionalization in development. In: Third World Quarterly, vol. 37, no. 12, pp. 2156-2157.

OECD (2018): Aid to gender equality and women's empowerment: an overview. www.oecd. org/dac/gender-development (19.3.2021).

Okech, Awino; Musindarwezo, Dinah (2019): Transnational feminism and the Post-2015 Development Agenda. In: Soundings, vol. 71, pp. 75-90.

Parpart, Jane L. (2014): Exploring the transformative potential of gender mainstreaming in international development institutions. In: Journal of International Development, vol. 26, pp. 382-395.

Rao, Sheila; Tiessen, Rebecca (2020): Whose feminism(s)? Overseas partner organizations' perceptions of Canada's Feminist International Assistance Policy. In: International Journal, vol. 75 , no. 3 , pp. 349-366.

Rauh, Karen (2010): NGOs, foreign donors, and organizational processes: Passive NGO recipients or strategic actors? In: McGill Sociological Review, vol. 1, pp. 29-45.

Robinson, Fiona (2021): Feminist foreign policy as ethical foreign policy? A care of ethics perspective. In: Journal of International Political Theory, vol. 17, no. 1, pp. 20-37.

Sundstrom, Lisa McIntosh (2005): Foreign assistance, international norms, and NGO development: Lessons from the Russian campaign. In: International Organization, vol. 59, no. 2, pp. 419-449.

Tiessen, Rebecca (2019): What's new about the Feminist International Assistance Policy: The problem and possibilities of "More of the Same". In: University of Calgary School of Public Policy Publications, vol. 12, no. 44, pp. 1-15.

Tiessen, Rebecca; Lough, Benjamin J.; Cheung, Samuel (2018): Introduction: A theoretical and methodological case for examining agency and power relations in North-South volunteering research collaborations. In: Tiessen, Rebecca; Lough, Benjamin J.; Grantham, Kate (ed.): Insights on International Volunteering. Perspectives From the Global South. Germany, pp. 7-12.

Win, Everjoice J. (2004): Not very poor, powerless or pregnant: The African woman forgotten by development. In: Institute Development Studies Bulletin, vol. 35, no. 4, pp. 61-64. 